\title{
The hard X-ray emission from the complex SNR MSH 15-52 observed by BeppoSAX
}

\author{
T. Mineo ${ }^{1}$, G. Cusumano ${ }^{1}$, M. C. Maccarone ${ }^{1}$, S. Massaglia $^{2}$, E. Massaro ${ }^{3, \dagger}$, and E. Trussoni ${ }^{4}$ \\ 1 Istituto di Fisica Cosmica con Applicazioni all'Informatica, CNR, via Ugo La Malfa 153, 90146 Palermo, Italy \\ 2 Dipartimento di Fisica Generale dell'Università, via Pietro Giuria 1, 10125 Torino, Italy \\ 3 Istituto di Astrofisica Spaziale, CNR, via Fosso del Cavaliere, 00113 Roma, Italy \\ 4 Osservatorio Astronomico di Torino, Strada Osservatorio 20, 10025 Pino Torinese, Italy
}

Received 8 August 2001 / Accepted 18 October 2001

\begin{abstract}
We present the results of a BeppoSAX observation of the Supernova Remnant MSH 15-52, associated with the pulsar PSR B1509-58, and discuss its main morphological and spectroscopic properties in the 1.6-200 keV energy range (MECS and PDS instruments). The two main structures of the remnant, the Southern Nebula, the plerion centered on the pulsar, and the Northern Nebula, are clearly visible in the MECS, with the former showing a much a harder spectrum. Furthermore, a diffuse extended emission surrounds the whole remnant up to $\approx 17^{\prime}$ from the center. Non-thermal flux is detected in the PDS up to $200 \mathrm{keV}$ as well, and it appears that also in this energy range the emission is not concentrated in the central region around the pulsar. These data imply that the plerion extends up to a few tens of parsecs from the pulsar.
\end{abstract}

Key words. ISM: individual objects: MSH 15-52 - ISM: supernova remnants - X-rays: ISM

\section{Introduction}

The source MSH 15-52 (G320.4-1.2), associated with the pulsar PSR B1509-58, is one of the most studied objects in the wide class of Supernova Remnants (SNR). The morphology of this remnant is quite complex: in the radio band it appears basically spherically symmetric (size $\approx 30^{\prime}$ ), typical of shell-like SNR, with bright spots in the NW and SE regions (see e.g. Milne et al. 1993, and references therein). The NW region coincides with the IR and optical nebula RCW 89, where irregular filaments are present, emitting in the $\mathrm{H}_{\alpha}$ line (Seward et al. 1983). Caswell et al. (1975) estimated a distance of $\approx 4.2 \mathrm{kpc}$ and the classical relationship between the surface brightness at $1 \mathrm{GHz}$ and the linear diameter $(\Sigma-D$ relation) yields an age of $\sim 1 \div 2 \times 10^{4}$ years (Clark \& Caswell 1976). At X-ray energies, diffuse emission is detected from the shell-like remnant with a bright spot coincident with the NW zone. Centered at the pulsar position, a plerion-like structure is also present with size similar to that of the northern region (see e.g. Trussoni et al. 1996, and references therein).

The pulsar has a period of $\approx 150 \mathrm{~ms}$ with one of the largest period derivatives, $\approx 1.5 \times 10^{-12} \mathrm{~s} \mathrm{~s}^{-1}$, and emits

Send offprint requests to: T. Mineo,

e-mail: mineo@ifcai.pa.cnr.it

† On leave from Università La Sapienza, Roma, Italy. at radio frequencies, in the soft and hard X-ray bands and at $\gamma$ energies (see e.g. Trussoni et al. 1990; Kawai et al. 1993; Matz et al. 1994; Greiveldinger et al. 1995; Rots et al. 1998). The spin-down age of $\approx 1700$ years, younger than that estimated for the SNR, suggested possible associations with the supernova that exploded in $185 \mathrm{AD}$ (Thorsett 1992). It was debated for a long time whether PSR B1509-58 and its surrounding plerion were components of the same system or just aligned along the line of sight. This apparent discrepancy can be reconciled assuming that the supernova exploded in an underdense cavity (Bhattacharya 1990). This possibility has been confirmed by the detailed radio observations of Gaensler et al. (1999).

Concerning the X-ray observations, several timing and spectroscopic data for the pulsar are available from the soft to the hard band. Spectroscopic and morphological data are necessary for the analysis of the properties of the remnant. So far, information on the structure of the remnant are available the in the low energy X-ray band (Einstein and ROSAT), up to $10 \mathrm{keV}$ by the observations of EXOSAT and ASCA and at very high energies (up to $250 \mathrm{keV}$ ) by the Ginga and RXTE missions.

In order to better understand the physics of these peculiar objects we observed MSH 15-52 and PSR B1509-58 with BeppoSAX in February 1998. The instrumentation on board this satellite provides spectroscopic analysis in a 
very wide X-ray band $(0.1-200 \mathrm{keV})$ with a spatial resolution of $\approx 1.5^{\prime}$, up to energies of $10 \mathrm{keV}$. Here we present the results of the observation of the extended nebula, while the data on the pulsar emission are discussed in a separate paper (Cusumano et al. 2001). The main result of the present analysis is the discovery that the hard emission up to about $200 \mathrm{keV}$ very likely originates from the extended nebula and not only from the inner plerion around the pulsar. In Sect. 2 we summarize the main X-ray properties of MSH 15-52 as obtained in previous missions, in Sect. 3 we outline the details of the observations and of the procedure for data reduction, in Sect. 4 we present the results of our analysis, while their astrophysical implications are discussed in Sect. 5 .

\section{The X-ray structure of $\mathrm{MSH} 15-52$ from previous observations}

From the images of the remnant obtained by ROSAT in the soft X-ray band (Fig. 1) we see the complex structure of MSH 15-52 (for details see Greiveldinger et al. 1995; Trussoni et al. 1996; Brazier \& Becker 1997). Around the pulsar position there is the plerion with quite uniform emission (Southern Nebula) and a cross-like shape, with the longest arms in the SE-NW direction (dimensions $\left.\approx 10^{\prime} \times 6^{\prime}\right)$. The northern arm merges into the Northern Nebula (coinciding with RCW 89), with dimensions $\approx 9^{\prime} \times 4^{\prime}$ at a distance of $\approx 7^{\prime}$ from the pulsar. In the HRI image the Northern Nebula shows an inhomogeneous morphology with several knots of emission (Brazier $\&$ Becker 1997). The opposite arm of the Southern Nebula ends in a curved tailed component with emitting blobs in the SE direction. All these structures are embedded in a diffuse and symmetric region, approximately centered at the pulsar position (Central Diffuse Nebula), of radius $\approx 15^{\prime}$. Outside this region there is a weak emission extended in the SE direction (Southern Extended Nebula) and coincident with the radio source G320.6-1.6 (Milne et al. 1993).

The X-ray flux from the Southern Nebula in the ROSAT band is consistent with a power law spectrum, with photon index $\Gamma \approx 2$ and column density $N_{\mathrm{H}}$ in the range $7 \div 9 \times 10^{21} \mathrm{~cm}^{-2}$. More complex is the case of the Northern Nebula: its PSPC spectrum fits with a thermal model with $k T \approx 0.4 \mathrm{keV}$ but with a column density about two times larger than in the Southern Nebula. However, ASCA observations of the Northern Nebula (Tamura et al. 1996) showed that the emission is consistent with a composite model: a thermal spectrum (in agreement with the PSPC results) plus a power law component, with $N_{\mathrm{H}} \approx 6 \times 10^{21} \mathrm{~cm}^{-2}$ and $\Gamma \approx 2$, similarly with the values found by the PSPC for the Southern Nebula. These results may be reasonably interpreted if the two arms in the NW-SE directions are opposite outflows emerging from the pulsar, while the shorter arms in the NE-SE direction could be related to an equatorial torus surrounding the pulsar, as proposed for the Crab Nebula (Hester et al. 1995; Brazier \& Becker 1997). Collimated winds

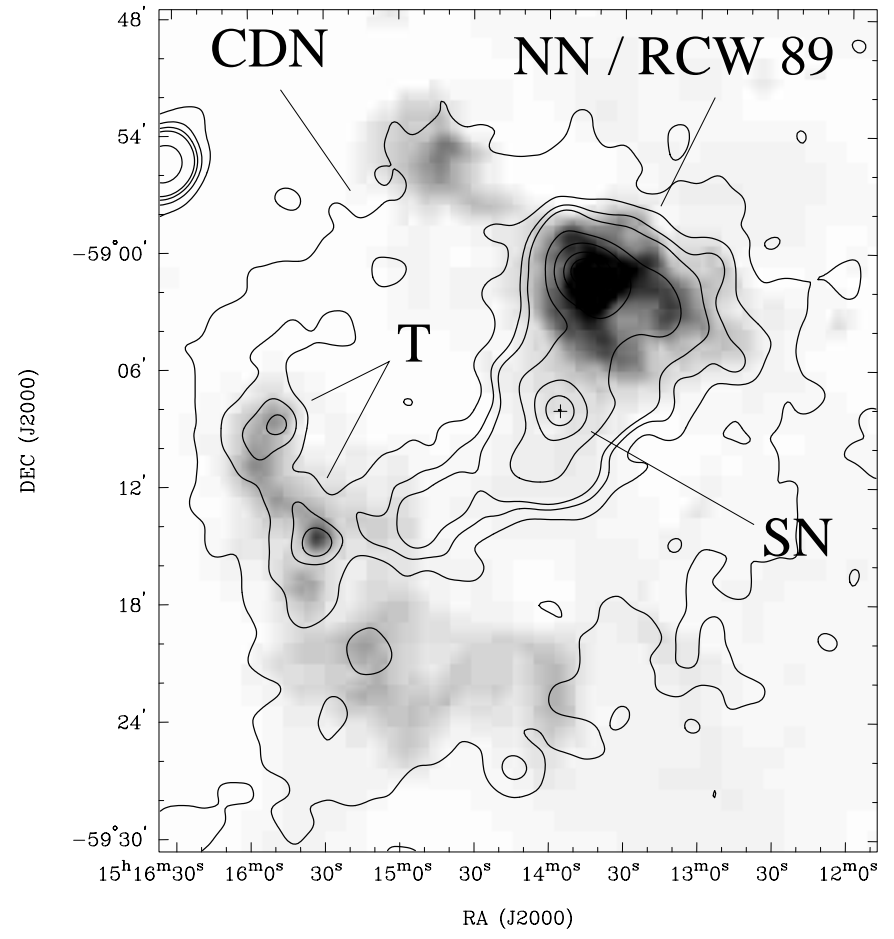

Fig. 1. Radio image of MSH 15-52 (36 cm) with the contour plot from the ROSAT PSPC observation $(0.1-2.4 \mathrm{keV})$. The cross marks the position of PSR B1509-58 (Gaensler et al. 1999). NN and SN indicate the Northern and Southern Nebulae respectively, $\mathrm{T}$ is the tail component and $\mathrm{CDN}$ the Central Diffuse Nebula.

accelerated from neutron stars have been proposed also to interpret the X-ray morphology of the Vela SNR (Markwardt \& Ögelman 1995), and were recently detected in a Chandra observation of the pulsar in the Crab Nebula (Weisskopf et al. 2000) and in the Vela SNR (Pavlov et al. 2001).

This picture of the remnant is further supported by the detailed radio observations of Gaensler et al. (1999) who suggested a strict interaction of the pulsar with the surrounding remnant. In particular, the X-ray knots detected in the Northern Nebula and in the tail coincide with analogous compact radio structures. These are interpreted as regions where the collimated outflows, originating from the pulsar, strongly interact with the environment. The positions of these radio/X-ray blobs in the Northern Nebula suggest that the direction of the jet axis changes with the time, following a sort of precession path. These radio data strongly support that, in spite of the apparent different ages, MSH 15-52 and PSR B1509-58 originated in the same event (conversely it is very likely that G320.6-016, coincident with the Southern Extended Nebula, is an older SNR along the same line of sight).

In harder X-ray bands, apart from ASCA, we have data only from non imaging instruments. The results from the observations of EXOSAT/ME (up to $11 \mathrm{keV}$, Trussoni et al. 1990), Ginga (up to $60 \mathrm{keV}$, Kawai et al. 1993) and RXTE (up to $250 \mathrm{keV}$, Marsden et al. 1997) confirm that also at these energies the spectrum follows a power law 


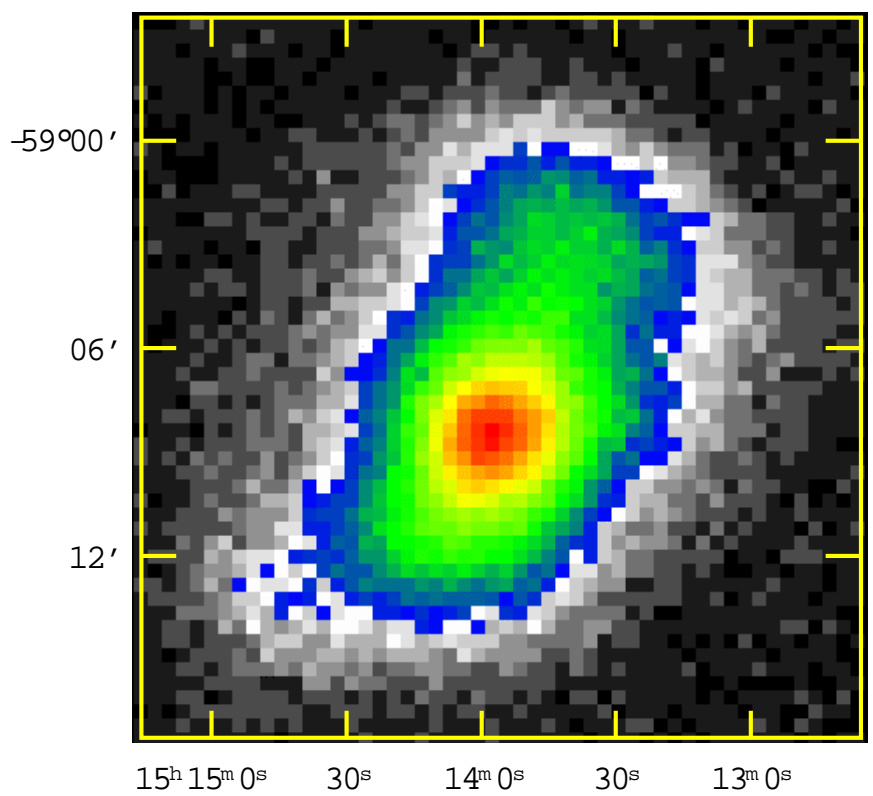

Fig. 2. Image of the complex SNR MSH 15-52 obtained by the MECS observation (1.6-10 keV). Spatial rebinning to a pixel size of $24 \times 24$ arcsec was applied. The position of the pulsar PSR B1509-58 coincides with the bright red spot at the center; this maximum corresponds to 1006 counts per pixel.

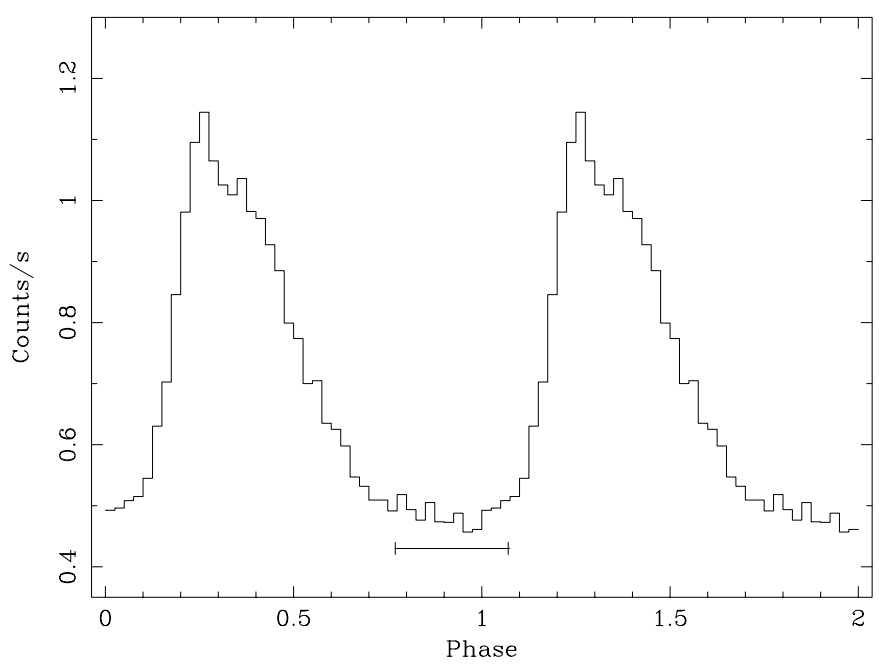

Fig. 3. Pulse profile of PSR B1509-58 in the $1.6-10 \mathrm{keV}$ energy band (MECS). The horizontal line corresponds to the phase interval used in the spectral analysis of the nebula.

with a photon index $\approx 2-2.2$, but the flux is about three times higher than deduced by the PSPC for the Southern Nebula. Furthermore, Marsden et al. (1997) found that a narrow iron line at $6.7 \mathrm{keV}$ significantly improved their fit.

\section{BeppoSAX observation and data analysis}

The BeppoSAX observation of MSH 15-52 was performed on 9-11 February 1998 for a total exposure of $31008 \mathrm{~s}$ in the LECS, $82483 \mathrm{~s}$ in the MECS and $34493 \mathrm{~s}$ in the PDS (for details on the BeppoSAX instrumentation see Boella et al. 1997). Standard procedures and selection criteria were applied to the data using the SAXDAS v.2.0.0 package.

A raw image of the remnant as it appears in the MECS (units 2 and 3) integrated over the entire energy band (1.6-10 keV) is shown in Fig. 2. The elongated structure in the SE-NW direction is clearly visible but the limited resolution does not allow one to resolve the minor substructures. It is possible to improve the image quality, up to the MECS spatial resolution, by applying a deconvolution algorithm based on the Lucy (1974) method to each monochromatic image and using the proper local Point Spread Function (PSF). The adopted procedure is described in detail in Maccarone et al. (2001). For each energy channel MECS images were first rebinned to a pixel size of $24 \times 24$ arcsec to improve the statistics, and then deconvolved with the proper monochromatic PSF. According to Maccarone et al. (2001) we used 300 iterations which guarantee a good convergence at all the energies. Final processed images were then added together in selected energy bands. In this analysis the pulsar contribution has not been eliminated to avoid strong reduction of the statistics.

To perform the spectral analysis of the nebular emission of the Southern Nebula we excluded the contribution from the pulsar. Phase histograms, for each energy channel of the NFIs instruments, have been obtained folding the selected UTC arrival times, converted to the Solar System Barycenter, with the pulsar ephemeris (see Cusumano et al. 2001 for detail). Figure 3 shows the resulting pulsed profile for the energy band $1.6-10 \mathrm{keV}$; the nebular emission has been extracted from the off-pulse phase interval (0.77-1.07) where we assume that the pulsar emission is not dominant. Spectral analysis was performed with the XSPEC package (ver. 11.0) after rebinning the counts in order to have at least 20 events in each energy channel. As the source is extended, a suitable auxiliary matrix must be created to correct for the vignetting, that is relevant at distances $\gtrsim 4^{\prime}$ from the center of the FOV (Fiore et al. 1999). This can be performed for the MECS data using the SAXDAS effarea command, but the procedure is suitable only for sources with symmetric azimuthal intensity distribution (for details see Molendi 1998; D'Acri et al. 1998). We then produced an ad-hoc matrix computing the vignetting correction according to the asymmetric spatial distribution of MSH 15-52. We then verified that using such auxiliary matrix the resulting best fit values of the spectral indices differ from those obtained with the standard matrices by less than $\approx 2 \sigma$ while the normalizations show a discrepancy of $\approx 30 \%$. No analogous procedure is available at the moment for the LECS observations of extended targets, therefore we have not included these data in our analysis.

Spectral fits were first performed separately for the MECS and PDS data and after a combined fit of both data sets was also considered. The spectrum of the background for the MECS has been extracted from blank fields, while the PDS one is simultaneously monitored by rocking the 

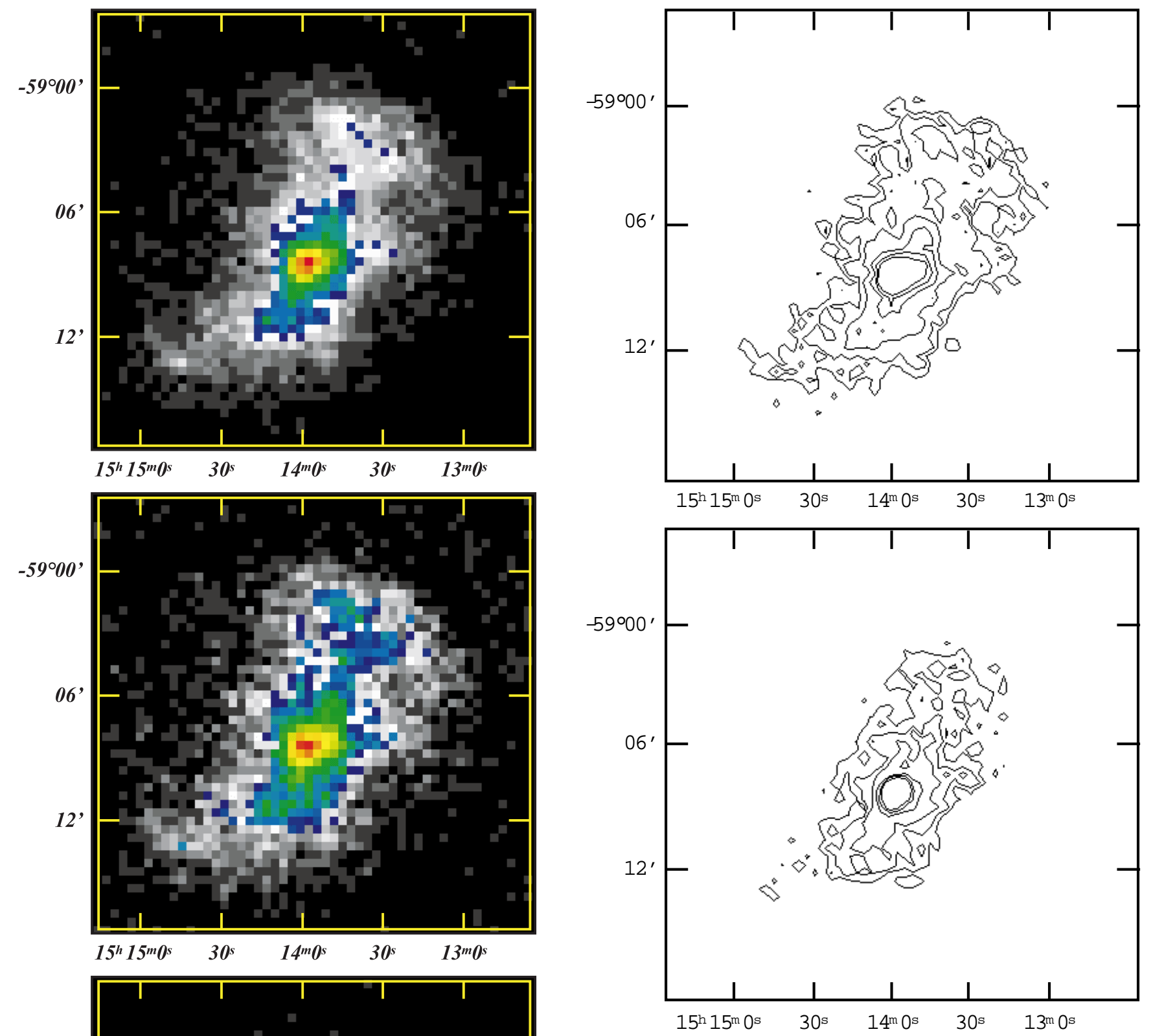

Fig. 5. Contour plots of the soft (1.6-4 keV, upper panel) and hard (4-10 keV, lower panel) deconvolved images of the Fig. 4. The contour levels correspond to the fractions of $1.00,0.75$, $0.50,0.25,0.10$, and 0.05 of the mean central intensity after the subtraction of the contribution from PSR B1509-58.

collimator. The uncertainties reported in the following are at 1 standard deviation for one interesting parameter.

\section{Results}

In this section we discuss first the remnant morphology in the intermediate band $(1.6-10 \mathrm{keV})$ as obtained from

Fig. 4. Deconvolved images of the SNR MSH 15-52 in the three energy bands: $1.6-10 \mathrm{keV}$ (upper panel), 1.6-4 keV (central panel), and $4-10 \mathrm{keV}$ (bottom panel). The maximum count levels are 4152, 1584, 2571 counts per pixel, respectively. the deconvolved MECS data, then we address the spectroscopic properties obtained from the MECS and PDS observations. 


\subsection{Morphology}

As expected from previous observations (see Sect. 2), the MECS image of this plerionic system shows an extended emission elongated at about the Position Angle $-20^{\circ}$, with a bright point source in the center corresponding to the pulsar position. The deconvolved images in three energy bands are shown in Fig. 4: 1.6-10 keV (upper panel), soft $\mathrm{X}$-ray band $1.6-4 \mathrm{keV}$ (central panel), hard X-ray band 4-10 keV (upper panel).

The contour plots of the last two images are also shown in Fig. 5. The contour levels are relative to the mean central intensity measured after subtraction of the contribution from the pulsar. In all these deconvolved images it is possible to recognize the major structures already known from ROSAT (Fig. 1). In particular, the much softer emission from the Northern Nebula is evident, that almost disappears for photon energies higher than $4 \mathrm{keV}$. In the harder band we notice the dominant central symmetric region, related to the pulsar emission, and the elongated structures towards the Northern Nebula and the tail region, respectively. This confirms the ASCA results (Tamura et al. 1996), that showed a highly asymmetric structure of the MSH 15-52 up to X-ray energies $\gtrsim 10 \mathrm{keV}$. The angular dimensions of the Southern Nebula in the higher energy range are at least $4^{\prime} \times 8.5^{\prime}$ (at the $10 \%$ level of the nebular central intensity), corresponding to about $5 \times 10 \mathrm{pc}$.

The soft spectrum of the Northern Nebula is also confirmed by the hardness ratio of these two images using pixel size of $2^{\prime} \times 2^{\prime}$ to improve the statistics (Fig. 6). The hardest value in the center of the nebula corresponds to the pulsar position, as expected.

We remark finally that due to the smaller FOV of the MECS, from the image we cannot disentangle the presence of the large Central Diffuse Nebula detected in the ROSAT observation.

\subsection{The spectral properties of the plerionic emission}

In the following we first analyze separately the data from the MECS and the PDS and after we present the combined analysis.

\subsubsection{MECS data}

Due to the complex structure of the remnant, we performed spectral fits to flux measured in the MECS from three different regions. The first two are centered at the pulsar position with radii $4^{\prime}$, corresponding to the central region of the Southern Nebula, and $17^{\prime}$, to include the extended emission excluding however a sector containing the Northern Nebula; the third region is centered on the Northern Nebula with radius $3^{\prime}$. For the first two, events from the off-pulse phase interval are considered, while, for the third one, no phase selection was performed because the pulsar contamination at such a distance was estimated around 3\%. Spectral fits were computed for a power-law distribution and the resulting parameter values are reported in Table 1. From the fit to the Southern Nebula emission $\left(4^{\prime}\right)$ we have deduced for the column density $N_{\mathrm{H}}=(8.0 \pm 1.5) \times 10^{21} \mathrm{~cm}^{-2}$, consistent within $1 \sigma$ with the value deduced from Cusumano et al. (2001), $9.1 \times 10^{21} \mathrm{~cm}^{-2}$, that we have adopted in our analysis.

Southern Nebula and Central Diffuse Nebula. The spectral slopes (Table 1) are much steeper than the pulsar one that was found to be equal to $\approx 1.2$. The flux from the larger area, including also the Central Diffuse Nebula contribution, has a slightly steeper spectral distribution slope, while the $2-10 \mathrm{keV}$ energy flux is approximately two times larger. If we include in this fit also the sector with Northern Nebula, the spectrum is slightly steeper $(\Gamma=2.08 \pm 0.01)$ with a total $2-10 \mathrm{keV}$ flux of $1.2 \times 10^{-10} \mathrm{erg} \mathrm{cm}^{-2} \mathrm{~s}^{-1}$, i.e. $\approx 1.3$ times higher than in the previous case with the Northern Nebula excluded.

We have searched for possible line emissions at 6.4 and $6.7 \mathrm{keV}$ in the MECS spectrum extracted over the $17^{\prime}$ radius region and found no evidence of such features. The $6.7 \mathrm{keV}$ upper limit at $90 \%$ confidence level, of $1.2 \times$ $10^{-4}$ photons $\mathrm{cm}^{-2} \mathrm{~s}^{-1}$, about $30 \%$ less than the line flux derived from RXTE data (Marsden et al. 1997), is compatible with the Galactic ridge emission (Koyama et al. 1989).

Northern Nebula. It is known from the ASCA data (Tamura et al. 1996) that a thermal and a power-law components contribute to the total flux, with the former that largely prevails below $3 \mathrm{keV}$. In order to avoid the contamination from thermal photons, we fitted the MECS data for energies $\geq 4 \mathrm{keV}$ and found a photon index of 2.10, steeper than that found for Southern Nebula (Table 1). We estimate that this power-law component contributes for $\approx 25 \%$ to the total flux of the remnant in the $2-10 \mathrm{keV}$ band, as expected from the previous results on the Southern Nebula and Central Diffuse Nebula.

\subsubsection{PDS data}

The power-law fit of the unpulsed high energy flux detected in the PDS gave a poorly constrained photon index and a total flux of $1.2 \times 10^{-10} \mathrm{erg} \mathrm{cm}^{-2} \mathrm{~s}^{-1}$ in the 20 $200 \mathrm{keV}$ energy range (see Table 2). The slope is consistent with the extrapolation of the spectrum at lower energies obtained with the MECS, implying that energy losses do not crucially affect the emitting electrons up to energies of $200 \mathrm{keV}$. The lack of imaging information and the not enough constrained evaluation of the energy flux do not allow a definite answer to the question concerning the spatial extent of the region emitting at these energies.

\subsubsection{Combined MECS and PDS data}

A spectral analysis of the combined data sets from the MECS (with extraction radii of $4^{\prime}$ and $17^{\prime}$ ) and the PDS can provide information on the size of the region for the hard X-ray emission. Note that now the MECS spectrum 


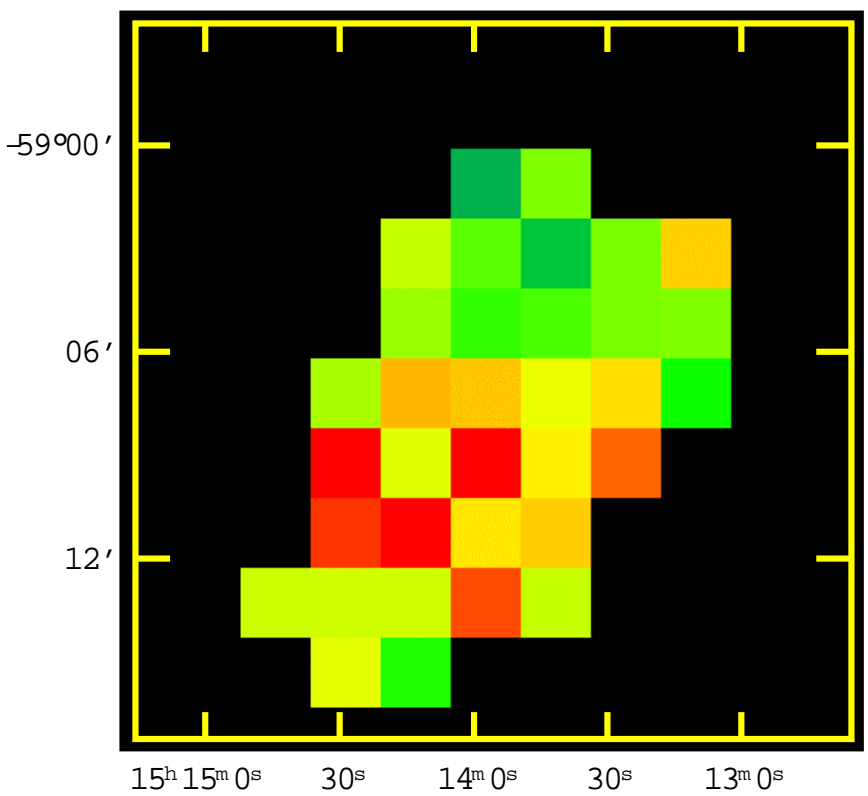

Fig. 6. Hardness ratio computed between the energy bands: 4$10 \mathrm{keV}, 1.6-4 \mathrm{keV}$. From Fig. 4 it is possible to see the location of the regions where the hardness ratio has been computed.

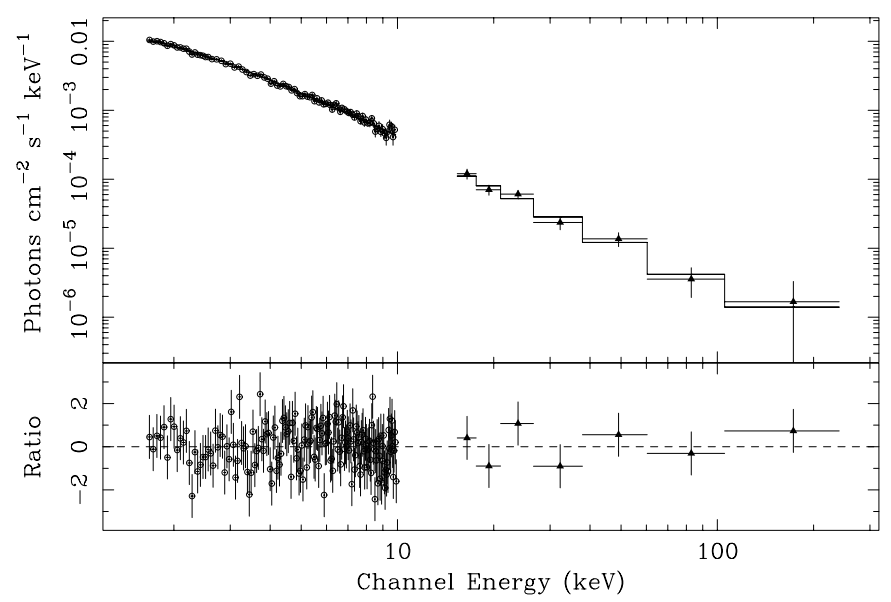

Fig. 7. MECS ( $17^{\prime}$ and including the Northern Nebula) and PDS spectrum of the whole nebula.

obtained with an extraction radius of $17^{\prime}$ includes the Northern Nebula contribution. When evaluating these fits, we considered as a free parameter the normalization factor $f_{\text {ic }}$ by which the MECS spectrum must be multiplied to match that from PDS. According to the in-flight calibrations with well-known sources, it is expected that $0.77 \lesssim f_{\text {ic }} \lesssim 0.95$ (Fiore et al. 1999), but this range applies only for point-like sources, while its value has not been calibrated for significantly extended sources, as in the present case. From the results reported in Table 2, we see that the values of this parameter are very different if the MECS data from the small or large region are considered. However, near the center of the FOV the vignetting effects are not relevant, as confirmed by our tests in Sect. 3, so that the Southern Nebula can be quite reasonably considered point-like in the MECS. Considering now the linear off-axis response of the PDS, if the region of emission of the hard photons is comparable (or smaller) than the Southern Nebula, we can assume again that it is "seen" basically point-like in the detector. Then, considering the hard component as the high energy tail of the emission detected in the MECS, and originating from the inner region of the plerion, we would expect $f_{\text {ic }}$ to lie in the range given above, from the fit of the MECS $\left(4^{\prime}\right)+$ PDS spectra. In contrast, we find $f_{\text {ic }} \approx 1.35$ (see Table 2), higher than the expected point source upper limit: this result implies that the MECS flux must be increased by $35 \%$ instead of being reduced about $15 \%$, as in the case of a point source. This discrepancy may be reasonably related to the fact that the region emitting in the $20-200 \mathrm{keV}$ is more extended than the Southern Nebula itself.

\section{Discussion}

The main results of this analysis concern the characteristics of the hard X-ray flux from the remnant: it turns out from the MECS + PDS data that the non-thermal unpulsed emission extends over the range 1.6-200 keV without any evidence of a break in the spectral slope. As far as the spatial emission distribution is concerned, in the range of the MECS it extends, without a strong break in the spectrum, up to $\gtrsim 17^{\prime}$ (i.e. $\gtrsim 20 \mathrm{pc}$ ) from the compact plerion that surrounds the pulsar. Furthermore there are some clues that also the emission up to $\approx 200 \mathrm{keV}$ may originate far from the central region.

Before discussing the implications of these data on the structure of MSH 15-52 one has to consider that $\mathrm{X}$-ray and $\gamma$-ray emission has been detected from the galactic ridge, where the remnant is located. In the energy range $2-11 \mathrm{keV}$ the average flux (consistent with a thermal continuum with temperature $\approx 9 \mathrm{keV}$ ) is $8.0 \times$ $10^{-8} \mathrm{erg} \mathrm{cm}^{-2} \mathrm{~s}^{-1} \mathrm{sr}^{-1}$ (Koyama et al. 1986), which is $\approx 20$ times less than the flux (for $\operatorname{arcmin}^{2}$ ) detected in the MECS. Regarding the hard range $(20-200 \mathrm{keV})$, a diffuse non-thermal emission from the galactic ridge has been detected, but with a much steeper slope than we obtain in the PDS ( $\Gamma \approx 2.7$, Skibo et al. 1997). Since no spurious hard source has been found in the field of view of the MECS, it is very reasonable that the hard spectrum is associated with the remnant and originates from a region more extended than the Southern Nebula.

The values of the spectral parameters are basically consistent with those derived, in the same range of energy, from the RXTE observation by Marsden et al. (1997) whose data were fitted by a spectrum with a slightly softer index $(\Gamma=2.21)$. However, in contrast with their conclusion, from the above arguments it is reasonable to conclude that the emission does not originate only from the inner plerion. Concerning the Northern Nebula, the slope of the spectrum is consistent with that deduced from ASCA within $1 \sigma$, while the flux at $1 \mathrm{keV}$ is $\approx 2$ times higher. Considering however the statistical errors the lower (in the MECS) and upper limits (in ASCA) of the fluxes differ by a factor $\approx 1.5$ and $\approx 1.2$ at $1 \sigma$ and $2 \sigma$ levels, respectively. 
Table 1. Power-law best fit parameters of the plerionic spectrum: MECS $(1.6-10 \mathrm{keV})$.

\begin{tabular}{llll}
\hline Parameter $^{\mathrm{a}}$ & MECS $\left(\mathrm{SN}^{\mathrm{e}}, 4^{\prime}\right)$ & $\mathrm{MECS}^{\mathrm{b}}\left(17^{\prime}\right)$ & MECS $\left(\mathrm{NN}, 3^{\prime}\right)$ \\
\hline Photon index $(\Gamma)$ & $1.90 \pm 0.02$ & $2.04 \pm 0.01$ & $2.10 \pm 0.06$ \\
Flux at $1 \mathrm{keV}^{\mathrm{c}}$ & $1.56 \pm 0.05$ & $3.68 \pm 0.07$ & $1.38 \pm 0.14$ \\
Energy flux & 0.47 & 0.89 & 0.31 \\
$\chi^{2}$ (d.o.f.) & $0.99(91)$ & $0.96(91)$ & $0.99(55)$ \\
\hline
\end{tabular}

${ }^{\text {a }} N_{\mathrm{H}}=9.1 \times 10^{21} \mathrm{~cm}^{-2}$;

b excluding a sector with the Northern Nebula (NN);

c $\times 10^{-2}$ photons $\mathrm{cm}^{-2} \mathrm{~s}^{-1} \mathrm{keV}^{-1}$;

d unabsorbed $\times 10^{-10} \mathrm{ergs}^{-2} \mathrm{sm}^{-1}, 2-10 \mathrm{keV}$ (MECS);

${ }^{\text {e }} \mathrm{SN}$ : Southern Nebula.

Table 2. Power-law best fit parameters of the plerionic spectrum: PDS (20-200 keV) and MECS + PDS.

\begin{tabular}{llll}
\hline Parameter $^{\mathrm{a}}$ & PDS & MECS $\left(\mathrm{SN}, 4^{\prime}\right)+\mathrm{PDS}$ & MECS $^{\mathrm{b}}\left(17^{\prime}\right)+\mathrm{PDS}$ \\
\hline Photon index $(\Gamma)^{2}$ & $2.1 \pm 0.2$ & $1.90 \pm 0.02$ & $2.08 \pm 0.01$ \\
Flux at $1 \mathrm{keV}^{\mathrm{c}}$ & $4.0 \pm 2.5$ & $1.57 \pm 0.0 .05$ & $5.16 \pm 0.09$ \\
Energy flux & 1.20 & & 1.37 \\
$f_{\text {ic }}$ & & $1.35 \pm 0.12$ & $0.73 \pm 0.06$ \\
$\chi^{2}$ (d.o.f.) & $0.68(6)$ & $0.87(98)$ & $1.04(98)$ \\
\hline
\end{tabular}

${ }^{\text {a }} N_{\mathrm{H}}=9.1 \times 10^{21} \mathrm{~cm}^{-2}$;

b including the Northern Nebula;

c $\times 10^{-2}$ photons $\mathrm{cm}^{-2} \mathrm{~s}^{-1} \mathrm{keV}^{-1}$;

d $\times 10^{-10} \mathrm{ergs} \mathrm{cm}^{-2} \mathrm{~s}^{-1}, 20-200 \mathrm{keV}$ (PDS).

The total luminosity of the remnant in the $2-10 \mathrm{keV}$ energy range is $2.6 \times 10^{35} \mathrm{erg} \mathrm{s}^{-1}$ (distance $=4.2 \mathrm{kpc}$ ) to which the Southern and Northern Nebulae contribute by $\approx 40 \%$ and $\approx 25 \%$, respectively. The remaining fraction of $\approx 35 \%$ comes from the extended region which is reasonable to associate with the Central Diffuse Nebula detected from ROSAT in the soft X-ray band. If the whole spectrum does not change up to $\sim 200 \mathrm{keV}$, the Northern Nebula and the Central Diffuse Nebula should contribute, by comparable amounts, to more than half of the flux detected in the PDS.

Hard X-ray emission is quite common in plerions and shell-like supernova remnants, which means that electrons of very high energy must be present. For the latter objects (like e.g. Cas A and SNR 1006) it is proposed that these particles are created through both the initial shock wave and local stochastic acceleration processes, and also a non thermal bremsstrahlung could be present (Reynolds 1996; Favata et al. 1997). In plerions conversely high energy electrons may originate from the pulsar and further be stochastically accelerated in the shock where the relativistic pulsar wind encounters the inner region of the remnant (Rees \& Gunn 1974; Kennel \& Coroniti 1984). A classical example of this picture is the Crab Nebula, where the shock should lie at $\sim 0.1 \mathrm{pc}$ from the pulsar, however the presence of a jet implies that the interaction of the pulsar outflow with the environment is anisotropic and very complex. We can reasonably imagine that a similar structure exists in the inner region of the Southern Nebula, with emission at high X-ray energies (notice its slightly flatter spectrum). The major problem in our case is the extension of the remnant, with $\approx 60 \%$ of the flux (up to $200 \mathrm{keV}$ ) originating several pc away from the inner plerion.

It is extremely difficult to describe the complicated high energy structure of MSH 15-52 through a detailed dynamic model of a pulsar relativistic wind interacting with a remnant. However we can try to see whether the radiating particles in the Central Diffuse Nebula may directly originate from the pulsar. In the simplest hypothesis, we can compare the Lorentz factor $\gamma$ of an electron which is synchrotron emitting a photon of energy $E$ in a magnetic field $B$ :

$\gamma \approx 4.5 \times 10^{8}\left(E_{\mathrm{keV}} / B_{\mu \mathrm{G}}\right)^{1 / 2}$,

$\left(E_{\mathrm{keV}}=E / 1 \mathrm{keV}\right.$ and $\left.B_{\mu \mathrm{G}}=B / 10^{-6} \mathrm{G}\right)$, with that of an electron originating from the outer gap region (Zhang \& Cheng 1997, Eq. (26)):

$\gamma_{\mathrm{og}} \approx 3 \times 10^{7} f^{1 / 2}\left(B_{12} R_{\star 6}^{3} / P\right)^{1 / 4}$, 
where $R_{\star 6}$ is the radius of the pulsar in units of $10^{6} \mathrm{~cm}$, $P$ is the period in s, $B_{12}$ the surface magnetic field in units of $10^{12} \mathrm{G}$ and $f$ a factor $\leq 1$ that takes into account the volume fraction of the outer gap in the magnetosphere. Assuming as reasonable value for the average nebular magnetic field $B=10 \mu \mathrm{G}$ (Seward et al. 1984) in Eq. (1) and the parameters of PSR B1509-58 in Eq. $(2)(P=0.15 \mathrm{~s}$, $B_{12}=15.5$ and $R_{\star 6}=1$ ), even in the extreme case of $f=1$ we find that $\gamma_{\mathrm{og}}$ is less by a factor $\approx 5$ and $\approx 15$ than required for the X-ray emission in the extended region at 10 and $100 \mathrm{keV}$, respectively.

The above reasoning is not entirely model-free, but relies upon theories of particle acceleration at the outer gap. However we can also draw similar conclusions if we postulate that an alternative process produces very high energy particles around the pulsar magnetosphere or in the inner region of the plerion. In fact a relativistic electron in a mean magnetic field, with a cooling time scale (neglecting other radiative losses):

$\tau=\gamma /|\dot{\gamma}|=1.7 \times 10^{12}\left(E_{\mathrm{keV}} B_{\mu \mathrm{G}}^{3}\right)^{-1 / 2} \mathrm{~s}$,

covers a distance:

$R \approx \kappa c \tau=1.6 \times 10^{4}\left(E_{\mathrm{keV}} B_{\mu \mathrm{G}}^{3}\right)^{-1 / 2} \mathrm{pc}$,

where through the factor $\kappa \leq 1$ we take into account that the particle cannot follow a pure radial propagation outwards. Practically we can consider $\kappa \sim R_{\mathrm{c}} / D$, i.e. the ratio of the electron gyroradius to the linear size of the remnant. With $B=10 \mu \mathrm{G}$ the maximum distance attained by the particle is $R \approx 50 \kappa$ pc for $E=100 \mathrm{keV}$ photons. Consistently with the extent of the Central Diffuse Nebula, one would require $\kappa \sim 0.5$, while the actual value of the electron gyroradius for a $100 \mathrm{keV}$ emitting electron is $\sim 2 \times 10^{11} \mathrm{~cm}$, implying a value of $\kappa \lesssim 10^{-8}$.

Then, excluding that the Central Diffuse Nebula is an old SNR on the line of sight and just overlapped and centered on the pulsar, it appears that in this remnant effective reacceleration processes must be effective far from the central plerion.

Coming to the Northern Nebula, the limited imaging capabilities of the MECS do not allow us to check whether this power law emission is related to the jet emerging from the pulsar (as proposed by Tamura et al. 1996) or to some region of local particle acceleration. In the former case in the collimated wind highly relativistic electrons must be present, and we find again the problem of the origin of such particles discussed above. If most of the hard emission is related to local acceleration (e.g. shocks), we could argue that a fraction of high energy particles might diffuse away from the Northern Nebula and contribute to the emission of the Central Diffuse Nebula. The detailed radio images of the Northern Nebula obtained by Gaensler et al. (1998) show discrete highly polarized spots coincident with the X-rays knots detected in the ROSAT/HRI image (Brazier \& Becker 1997). These structures, whose emission is consistent with a single power law from radio to X-ray energies, could be interpreted as shocks due to the interaction of a precessing jet with the environment. It is possible to see that the total X-ray emission from these knots is about twice that deduced from the ASCA observations, but more consistent with the MECS results. In conclusion, the physical conditions of the emitting plasma in the Northern Nebula are quite complex, and only detailed observations from the new generation satellites will contribute new insights on this point.

Acknowledgements. The authors are grateful to F. Fiore for his information on the analysis of extended images. ET and SM acknowledge financial support from the Agenzia Spaziale Italiana through the grant ASI ARS 99 15. EM acknowledges financial support from the Italian Ministry of University and Scientific Research through the grant Cofin 99-02-02.

\section{References}

Bhattacharya, D. 1990, JApA, 11, 125

Boella, G., Butler, R. C., Perola, G. C., et al. 1997, A\&AS, 122,299

Brazier, K. T. S., \& Becker. W. 1997, MNRAS, 284, 335

Caswell, J. L., Murray, J. D., Roger, R. S., Cole, D. J., \& Cooke, D. J. 1975, A\&A, 45, 239

Clark, D. H., \& Caswell, J. L. 1976, MNRAS, 174, 267

Cusumano, G., Mineo, T., Massaro, E., et al. 2001, A\&A, 375, 397

D’Acri, F., de Grandi, S., \& Molendi, S. 1998, in The Active Xray Sky: Results from BeppoSAX and RXTE, ed. L. Scarsi, H. Bradt, P. Giommi, \& F. Fiore, Nucl. Phys. B, Proc. Suppl., 69, 581

Favata, F., Vink, J., Dal Fiume, D., et al. 1997, A\&A, 324, L49

Fiore, F., Guainazzi, M., \& Grandi, P. 1999 in Cookbook for BeppoSAX NFI Spectral Analysis, BeppoSAX Science Data Center, version 1.2

Gaensler, B. M., Brazier, K. T. S, Manchester, R. N, Johonston, S., \& Green, A. J. 1999, MNRAS, 305, 724

Greiveldinger, S., Caucino, C., Massaglia, S., Ögelman, H., \& Trussoni, E. 1995, ApJ, 454, 855

Hester, J. J., Scowen, P. A., Sankrit, R., et al. 1995, ApJ, 448, 240

Kawai, N., Okayasu, R., \& Sekimoto, Y. 1993, in Compton Gamma-ray Observatory, ed. M. Friedlander, N. Gehrels, \& D. J. Macomb (AIP, New York), AIP Conf. Proc., 280, 213

Kennel, C. F., \& Coroniti, F. V. 1984, ApJ, 283, 710

Koyama, K., Makishima, K., Tanaka, Y., \& Tsunemi, H. 1986, PASJ, 38, 121

Lucy, L. B. 1974, AJ, 79, 745

Maccarone, M. C., Mineo, T., \& Preite-Martinez, A. 2001, A\&A, 368, 267

Markwardt, C. B., \& Ogelman H. 1995, Nature, 375, 40

Marsden, D., Blanco, P. R., Gruber, D. E., et al. 1997, ApJ, 491, L39

Matz, S. M., Ulmer, M. P., Grabelsky, D. A., et al. 1994, ApJ, 434,288

Milne, D. K., Caswell, J. L., \& Haynes, R. F. 1993, MNRAS, 264, 853 
Molendi, S. 1998 in The Active X-ray Sky: Results from BeppoSAX and RXTE, ed. L. Scarsi, H. Bradt, P. Giommi, \& F. Fiore, Nucl. Phys. B, Proc. Suppl., 69, 563

Pavlov, G. G., Kargaltsev, O. Y., Sanwal, D., \& Garmire, G. P. 2001, ApJ, 554, L189

Rees, M. J., \& Gunn, J. E. 1974, MNRAS, 167, 1

Reynolds, S. P. 1996, ApJ, 459, L13

Rots, A. H., Jahoda, K., Macomb, D. J., et al. 1998, ApJ, 501, 749

Seward, F. D., Harnden, F. R. Jr., Murdin, P., \& Clark D. H. 1983, ApJ, 267, 698

Seward, F. D., Harnden, F. R. Jr., Szymkowiak, A., \& Swank, J. 1984, ApJ, 281, 650
Skibo, J. G., Johnson W. N., Kurfess, J. D., et al. 1997, ApJ, 483, L95

Tamura, K., Kawai, N., Yoshida, A., \& Brinkmann, W. 1996, PASJ, 48, 33

Thorsett, S. E. 1992, Nature, 356, 690

Trussoni, E., Brinkmann, W., Ögelman, H., et al. 1990, A\&A, 234,403

Trussoni, E., Massaglia, S., Caucino, S., Brinkmann, W., \& Aschenbach, B. 1996, A\&A, 306, 581

Weisskopf, M. C., Hester, J. J. Tennant, A. E., et al. 2000, ApJ, 536, L81

Zhang, L., \& Cheng, K. S. 1997, ApJ, 487, 370 